\title{
Maintaining focus on administering effective malaria treatment during the COVID-19 pandemic
}

\author{
J Raman, ${ }^{1,2,3} \mathrm{PhD}$; K I Barnes, ${ }^{4,5} \mathrm{MB}$ ChB, MMed (Clin Pharmacol); L Baker, ${ }^{6}$ Dip Pharm; M Blaylock, ${ }^{7} \mathrm{MB}$ ChB; \\ L Blumberg, ${ }^{1,8}$ MB BCh, MMed (Micro), DTM\&H, DOH, DCH; J Frean, ${ }^{1,2}$ MB ChB, MMed (Path Microbiol); E Misiani, ${ }^{9} \mathrm{PhD}, \mathrm{MPH}$; \\ I S Ukpe, ${ }^{10,11} \mathrm{MB} B C h$, MMed (Fam Med) \\ ${ }^{1}$ Centre for Emerging Zoonotic and Parasitic Diseases, National Institute for Communicable Diseases, National Health Laboratory Service, \\ Johannesburg, South Africa \\ ${ }^{2}$ Wits Research Institute for Malaria, School of Pathology, Faculty of Health Sciences, University of the Witwatersrand, Johannesburg, South Africa \\ ${ }^{3}$ University of Pretoria Institute for Sustainable Malaria Control, School of Health Systems and Public Health, University of Pretoria, South Africa \\ ${ }^{4}$ Division of Clinical Pharmacology, Department of Medicine, Faculty of Health Sciences, University of Cape Town, South Africa \\ ${ }^{5}$ WorldWide Antimalarial Resistance Network (WWARN), Division of Pharmacology, Faculty of Health Sciences, University of Cape Town, \\ South Africa \\ ${ }^{6}$ Amayeza Info Services, Johannesburg, South Africa \\ ${ }^{7}$ KwaZulu-Natal Provincial Department of Health, Manguzi, South Africa \\ ${ }^{8}$ Faculty of Veterinary Medicine, University of Pretoria, South Africa \\ ${ }^{9}$ Malaria and Other Vector-Borne and Zoonotic Diseases Directorate, National Department of Health, Pretoria, South Africa \\ ${ }^{10}$ Mpumalanga Provincial Department of Health, Mbombela, South Africa \\ ${ }^{11}$ Department of Family Medicine, Faculty of Health Sciences, University of Pretoria, South Africa
}

Corresponding author: J Raman (jaishreer@nicd.ac.za)

\begin{abstract}
As September marks the start of the malaria season in South Africa (SA), it is essential that healthcare professionals consider both COVID19 and malaria when a patient who lives in or has recently travelled to a malaria area presents with acute febrile illness. Early diagnosis of malaria by either a rapid diagnostic test or microscopy enables prompt treatment with the effective antimalarial, artemether-lumefantrine, preventing progression to severe disease and death. Intravenous artesunate is the preferred treatment for severe malaria in both children and adults. Adding single low-dose primaquine to standard treatment is recommended in endemic areas to block onward transmission. Use of the highly effective artemisinin-based therapies should be limited to the treatment of confirmed malaria infections, as there is no clinical evidence that these antimalarials can prevent or treat COVID-19. Routine malaria case management services must be sustained, in spite of COVID-19, to treat malaria effectively and support SA's malaria elimination efforts.
\end{abstract}

S Afr Med J 2021;111(1):13-16. https://doi.org/10.7196/SAMJ.2021.v111i1.15337

With the current COVID-19 pandemic stretching South Africa (SA)'s already severely overburdened health system to its limits, the prompt diagnosis and urgent treatment of malaria with the most effective therapies has become even more critical. Untreated uncomplicated malaria, particularly infections caused by Plasmodium falciparum in non-immune individuals, rapidly progresses to severe disease (requiring intensive care) and often death. As fever is the primary symptom used for the screening of both malaria and COVID-19 in SA, it is essential that healthcare workers maintain a high index of suspicion for malaria when a patient who lives in or has recently visited a malaria area presents with acute febrile illness. As SA enters the start of the malaria season (September to May), confirming or excluding a malaria infection when faced with such cases of acute febrile illness is now an imperative.

\section{Signs and symptoms}

Like those of many infectious diseases prevalent in Africa, the common early-stage clinical features of both malaria and COVID-19 are nonspecific and include fever, headaches, fatigue and sore joints/ muscles. In areas where there is a high likelihood of co-infection, confirmatory tests for both diseases should be conducted as soon as possible to inform treatment. In the wake of the COVID-
19 pandemic, many healthcare professionals may attribute acute respiratory distress syndrome (ARDS) solely to severe COVID-19 infection. However, it must be borne in mind that young children with malaria and patients with severe, complicated malaria often experience respiratory distress. This overlap in symptoms emphasises the importance of prompt testing for both diseases when patients living in malaria areas or who have recently travelled to a malaria risk area present with ARDS. Levels of certain biomarkers can assist with the differential diagnosis of these two diseases. COVID-19 patients often present with lymphopenia, ${ }^{[1]}$ while thrombocytopenia is common in malaria patients. ${ }^{[2,3]}$ Although there have been recent reports of raised bilirubin levels in hospitalised patients with COVID$19,{ }^{[4,5]}$ total bilirubin levels in malaria patients, particularly those with severe malaria, are significantly higher ${ }^{[6,7]}$ However, some other biochemical changes associated with COVID-19 are also shared with moderate to severe malaria, e.g. raised C-reactive $\operatorname{protein}^{[8,9]}$ and transaminases. ${ }^{[7,10]}$

\section{Point-of-care diagnosis}

Unlike COVID-19, malaria has sensitive antigen-based point-of-care diagnostics that are readily available across the public and private healthcare sectors in SA. Malaria rapid diagnostic tests (RDTs) 
generally produce a result within 20 minutes, enabling prompt treatment in the event of a positive test result or further investigation if the test is negative. As $>90 \%$ of the malaria infections in SA are caused by $P$. falciparum, a falciparum-specific, histidine-rich protein 2 (HRP2)-based type of RDT is preferred. While HRP2based RDTs are highly sensitive, they must not be used for follow-up monitoring of treated malaria patients, as they could continue to give a positive result for up to 30 days after the patient has completely recovered from the disease, owing to persistent circulation of the HRP2 ${ }^{[11]}$ For follow-up monitoring of a malaria patient, blood smear microscopy is the appropriate test. In line with recommendations from the World Health Organization (WHO), all patients should be followed up by microscopy on day 28 to confirm parasite clearance. Microscopic examination of blood smears is also required to quantify parasite density in order to diagnose hyperparasitaemia $(>4 \%$ or $3+$ parasitaemia), which should be treated as severe malaria.

\section{Uncomplicated malaria}

Despite an increasing prevalence of falciparum parasites resistant to the recommended antimalarial treatment, artemisinin-based combination therapies (ACTs), in the greater Mekong subregion of South-East Asia ${ }^{[12]}$ and in Rwanda in Africa, ${ }^{[13]}$ the ACT first introduced into KwaZulu-Natal Province in 2001, ${ }^{[14]}$ artemetherlumefantrine, remains highly efficacious in SA. ${ }^{[15]}$ However, efficacy hinges on ensuring the correct dosage (Table 1), completion of the full treatment course, and ensuring that every dose is taken with at least $1.2 \mathrm{~g}$ of fat for optimal absorption of lumefantrine. ${ }^{[16]}$ Currently in SA, artemether-lumefantrine, marketed as Coartem, is registered for use in patients weighing $<65 \mathrm{~kg}$, with the Coartem product information leaflet stating that 'No adequate experience has been acquired in the use of $\mathrm{Coartem}^{\circ}$ in patients weighing more than $65 \mathrm{~kg}$.' As there have been suggestions that patients weighing $>85 \mathrm{~kg}$ are more susceptible to treatment failure, it is critical to ensure full adherence and fat co-administration in this cohort of patients. For patients weighing $>85 \mathrm{~kg}$, extending the treatment course to FIVE days is advised, administering FOUR tablets per dose, given twice daily for a total of 10 doses (off-label recommendation).

In addition, it should be noted that some population groups that are often excluded from clinical trials, such as pregnant women and infants, those with comorbidities (e.g. malnutrition) and/or those taking concomitant medication (e.g. efavirenz, rifampicin), may be at increased risk of suboptimal antimalarial exposure and a poorer response to treatment. ${ }^{[17-20]}$ Particularly careful attention should therefore be paid to ensuring their full adherence and monitoring their response to treatment. The use of oral quinine for the treatment of uncomplicated malaria is strongly discouraged, and it should only be used in patients allergic to artemether-lumefantrine.

While ACTs are primarily used to treat falciparum infections, they are highly effective against asexual blood stages of non- falciparum species as well. Non-falciparum infections should be treated with artemether-lumefantrine as for falciparum infections (Table 1). A 14-day course of standard-dose primaquine phosphate (15 mg/d for adults or $0.25-0.5 \mathrm{mg}$ base $/ \mathrm{kg} / \mathrm{d}$ ) should follow artemether-lumefantrine treatment in children and adults with relapsing malaria ( $P$. vivax or $P$. ovale), including mixed infections. However, primaquine should not be given to infants or to women who are pregnant and/or breastfeeding. Where available, glucose-6phosphate dehydrogenase (G6PD) status should guide administration of primaquine to prevent relapse. In individuals with confirmed G6PD deficiency, administration of primaquine base at $0.75 \mathrm{mg} /$ $\mathrm{kg}$ once a week for 8 weeks, with close medical supervision for potential primaquine-induced adverse haematological effects, is recommended.

Based on the gametocytocidal (transmission-blocking) properties of the antimalarial primaquine, the WHO recommended adding a single low dose of primaquine to ACTs to assist countries attempting to eliminate malaria as well as halt spread of artemisininresistant parasites. ${ }^{[21]}$ Unlike the higher doses and longer courses of primaquine required for the radical cure of $P$. vivax and $P$. ovale malaria, the single low dose of primaquine recommended for transmission blocking is not associated with severe haemolysis in G6PD-deficient individuals. ${ }^{[22]}$ Following local data that confirmed the safety of single low-dose primaquine, ${ }^{[15]}$ the South African Health Products Regulatory Authority approved its use under a section 21A application in selected health facilities in low-transmission malaria endemic districts to support malaria elimination efforts in SA.

\section{Severe malaria}

Patients with severe malaria present with a range of clinical signs and symptoms (Table 2). If one (or more) of these features is present, the patient should be treated promptly with intravenous (IV) or intramuscular artesunate (Garsun), the recommended treatment for severe malaria in SA. Injectable artesunate was registered for use in SA in June 2017, so section 21 approval for its use to treat severe malaria is no longer required. Treatment outcomes with IV artesunate compared with IV quinine are significantly better in both adults and children, ${ }^{[23]}$ with fatality rates reduced by $39 \%$ in adults and $24 \%$ in children treated with IV artesunate as opposed to IV quinine. ${ }^{[24]}$ In addition, IV artesunate is more cost-effective, has a simpler dosage regimen (Table 3), is easier to administer, has a better safety profile, and requires no dosage adjustment in renal or hepatic impairment.

Patients with severe malaria should receive at least three doses of IV artesunate (at 0,12 and 24 hours), or the treatment should be continued until the patient is well enough to tolerate oral therapy, up to a maximum of 7 days with close monitoring for potential recrudescence. Once the patient is able to swallow, a complete full course (six doses) of artemether-lumefantrine as outlined in Table 1

Table 1. Dosing schedule of artemether-lumefantrine

\begin{tabular}{|c|c|c|c|c|c|c|}
\hline \multirow[b]{2}{*}{ Body weight } & \multicolumn{6}{|c|}{ Time of dose and number of tablets } \\
\hline & 0 hours & 8 - 12 hours & 24 hours & 36 hours & 48 hours & 60 hours \\
\hline $5-<15 \mathrm{~kg}$ & 1 tablet & 1 tablet & 1 tablet & 1 tablet & 1 tablet & 1 tablet \\
\hline $15-<25 \mathrm{~kg}$ & 2 tablets & 2 tablets & 2 tablets & 2 tablets & 2 tablets & 2 tablets \\
\hline $25-<35 \mathrm{~kg}$ & 3 tablets & 3 tablets & 3 tablets & 3 tablets & 3 tablets & 3 tablets \\
\hline $35-<65 \mathrm{~kg}$ & 4 tablets & 4 tablets & 4 tablets & 4 tablets & 4 tablets & 4 tablets \\
\hline$>65 \mathrm{~kg}^{*}$ & 4 tablets & 4 tablets & 4 tablets & 4 tablets & 4 tablets & 4 tablets \\
\hline
\end{tabular}

${ }^{*}$ For patients weighing $>85 \mathrm{~kg}$, extend treatment course to FIVE days, administering FOUR tablets per dose, given twice daily for a total of 10 doses (off-label recommendation). 


\section{Table 2. Clinical and laboratory features of severe malaria}

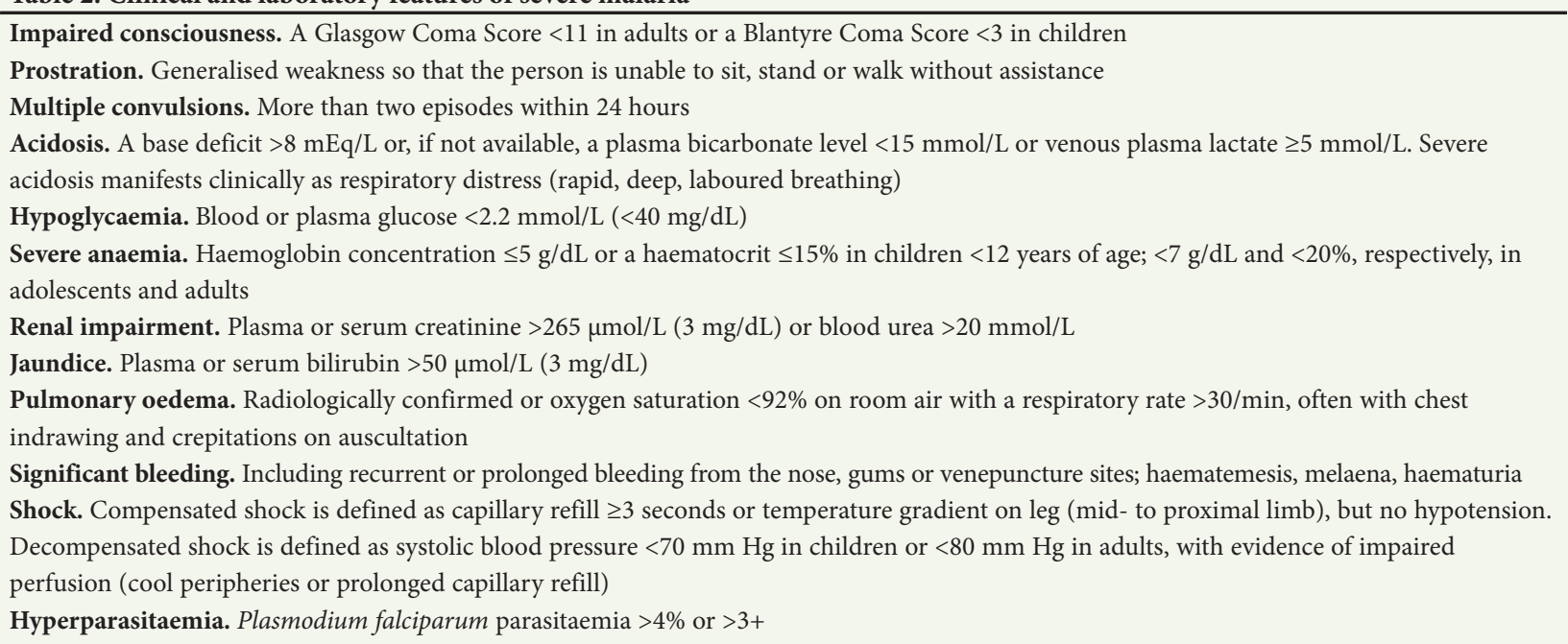

Table 3. Dosing schedule for intravenous artesunate

\begin{tabular}{ll}
\hline Body weight & Dosing \\
\hline Children $<20 \mathrm{~kg}$ & $\begin{array}{l}3 \mathrm{mg} / \mathrm{kg} \text { at } 0,12 \text { and } 24 \text { hours then daily until patient is able to tolerate } \\
\text { oral treatment, when full 6-dose 3-day artemether-lumefantrine treatment } \\
\text { should be given }\end{array}$ \\
$\begin{array}{l}2.4 \mathrm{mg} / \mathrm{kg} \text { at } 0,12 \text { and 24 hours then daily until patient is able to tolerate } \\
\text { oral treatment, when full 6-dose 3-day artemether-lumefantrine treatment } \\
\text { should be given }\end{array}$ &
\end{tabular}

must be administered. After IV artesunate, treatment-related delayed haemolysis may occasionally occur, ${ }^{[25]}$ so haemoglobin values of recovering patients, particularly non-immune patients and those with high parasite densities, should be closely monitored for up to 28 days.

Given the significant mortality reduction and cost-effective treatment advantages of IV artesunate, ${ }^{[23,24]}$ the use of IV quinine for the treatment of severe malaria at any healthcare facility, public or private, is strongly discouraged. Denying patients access to life-saving IV artesunate while depleting existing stocks of IV quinine could be considered unethical. Use of IV quinine should only be considered if either the patient is allergic to artesunate or IV artesunate is not immediately available at the health facility. In the rare instances that IV quinine has to be administered, a loading dose $(20 \mathrm{mg} / \mathrm{kg}$ body weight) must be given as a slow infusion (over 2 - 4 hours), followed by a maintenance dose $(10 \mathrm{mg} / \mathrm{kg}$ body weight) 8 hours later. Treatment should be administered every 8 hours until the patient is able to tolerate oral medication.

\section{Pregnant women}

As malaria in pregnancy is associated with low-birthweight infants, a heightened risk of anaemia, and an increased risk of severe malaria, pregnancy loss and mortality in low-transmission areas, ${ }^{[26]}$ it is critically important that malaria in pregnancy is treated as a medical emergency. For uncomplicated malaria, ACTs such as artemetherlumefantrine are associated with significantly better treatment outcomes than quinine in all trimesters, ${ }^{[27]}$ and are therefore the recommended treatment in SA. ${ }^{[28]}$ Severe malaria in pregnancy should be preferentially treated with IV artesunate, as pregnant women are at significantly increased risk of quinine-associated hypoglycaemia. ${ }^{[29]}$

\section{COVID-19 co-infection}

Currently there are very limited data on treatment outcomes of malaria patients co-infected with COVID-19. In patients with a mild COVID-19 infection and uncomplicated malaria, we recommend that the standard weight-based six-dose artemether-lumefantrine treatment (Table 1) be administered, with the patient closely monitored to ensure complete parasite clearance. In critically ill patients, IV artesunate should be administered as soon as possible, with parasitaemia levels regularly monitored by microscopy to assess treatment response.

Chloroquine or hydroxychloroquine use should be limited to treatment of inflammatory autoimmune diseases such as systemic lupus erythematosus (SLE) and rheumatoid arthritis (RA). ${ }^{[30-32]}$ Although initial in vitro data showed that hydroxychloroquine was capable of effectively inhibiting COVID-19, ${ }^{[33,34]}$ these findings have not been replicated in in vivo clinical trial settings. The administration of hydroxychloroquine to patients with mild COVID-19 symptoms did not reduce viral load or the risk of hospitalisation, or shorten the time to complete resolution of symptoms, compared with individuals who received the standard-of-care treatment. ${ }^{[35]}$ In patients with severe COVID-19, hydroxychloroquine administration did not affect intubation or in-hospital mortality rates, ${ }^{[36,37]}$ and was associated with a prolonged QT interval. ${ }^{[38]}$ In addition, the prophylactic effect of hydroxychloroquine against COVID-19 in individuals with SLE and RA has been shown to be negligible. ${ }^{[39]}$ Although chloroquine was previously very effective against falciparum malaria and had a good safety profile, ${ }^{[40]}$ the widespread entrenchment of chloroquine-resistant falciparum parasites rendered the antimalarial ineffective on the African continent and beyond, ${ }^{[41]}$ necessitating its replacement with ACTs.

Because artemisinin and its derivatives have anti-inflammatory and immune regulatory properties, ${ }^{[42]}$ there have been suggestions 
that these drugs can be used to treat COVID-19 infections. However, at present there is no clinical evidence to support any use of artemisinin or any related herbal compounds in either the prevention or the treatment of COVID-19. Use of artemisinin and its derivatives must therefore be restricted to the treatment of malaria infections.

\section{Conclusions}

To ensure that the current COVID-19 pandemic does not result in a major upsurge in malaria cases, derailing SA's elimination efforts, it is critical that the recommended malaria case management practices and procedures are closely adhered to by all healthcare practitioners. In addition, artemisinin-based therapies must be reserved for the treatment of malaria, as they are the only class of antimalarial still effective against falciparum malaria and have no proven effect in either preventing or treating COVID-19.

\section{Declaration. None.}

Acknowledgements. None.

Author contributions. JR drafted the manuscript and collated the input provided by KIB, LBa, MB, LBl, JF, EM and ISU. KIB and JF assisted with editing. All authors approved the submission of the final version.

Funding. None.

Conflicts of interest. None.

1. Huang I, Pranata R. Lymphopenia in severe coronavirus disease-2019 (COVID-19): Systematic review and meta-analysis. J Intensive Care 2020;8:36. https://doi.org/10.1186/s40560-020-00453-4

2. D'Acremont V, Landry R, Mueller I, et al. Clinical and laboratory predictors of imported malaria in an outpatient setting: An aid to medical decision making in returning travellers with fever. Am J Trop Med Hyg 2000;66(5):481-486. https://doi.org/10.4269/ajtmh. 2002.66.481

Hyg 2000;66(5):481-486. https:///doi.org/10.4269/ajtmh.2002.66.481
3. Lacerda MVG, Maurao MPG, Coelho HCC, et al. Thrombocytopenia in malaria: Who cares? Mem Inst Lacerda MVG, Maurao MPG, Coelho HCC, et al. Thrombocytopenia in malaria: Who cares?
Oswaldo Cruz 2011;106(Suppl 1):52-63. https://doi.org10.1590/s0074-02762011000900007

4. Hundt MA, Deng Y, Ciarleglio MM, et al. Abnormal liver tests in COVID-19: A retrospective observation 4. Hundt MA, Deng Y, Ciarleglio MM, et al. Abnormal liver tests in COVID-19: A retrospective observation
cohort study of 1827 patients in a malaria US hospital network. Hepatology 2020;72(4):1169-1176. cohort study of 1827 patients in
https://doi.org/10.1002/hep.31487

5. Paliogiannis P, Zinellu A. Bilirubin levels in patients with mild and severe COVID-19: A pooled analysis. Liver Int 2020;40(7):1787-1788. https://doi.org/10.1111/liv.14477

6. Mphahlele BJ, Mpe MJ. Falciparum malaria in a South African tertiary care hospital. Pol Arch Med Wewn 2008;118(6):351-355. https://doi.org/10.20452/pamw.412

7. Ramirez J, Porras B, Borrero E, et al. Factors associated with the severity and complications of patients with malaria hospitalized between 2009 and 2013 in three municipalities of Colombia: Case control study. Malar J 2016;15:514. https://doi.org/10.1186/s12936-016-1554-5

8. Liu F, Li L, Xu M, et al. Prognostic value of interleukin-6, C-reactive protein, and procalcitonin in patients with COVID-19. J Clin Virol 2020;127:104370. https://doi.org/10.1016/j.jcv.2020.104370

9. Bhardwaj N, Ahmed MZ, Sharma S, et al. C-reactive protein as a prognostic marker of Plasmodium falciparum malaria severity. J Vector Borne Dis 2019;56(2):1220126. https://doi.org/10.4103/0972falciparum

10. Tian W, Jian W, Yao J, et al. Predictors of mortality in hospitalized COVID-19 patients: A systematic review and meta-analysis. J Med Virol 2020;92(10):1875-1883. https://doi.org/10.1002/jmv.26050

11. Iqbal J, Siddique A, Jameel M, et al. Persistent histidine-rich protein 2, parasite lactate dehydrogenase and panmalarial antigen reactivity after clearance of Plasmodium falciparum monoinfection. J Clin Microbio 2004;42(9):4237-4241 https://doi.org/10.1128/JCM.42.9.4237-4241.2004

12. Ashley EA, Dhorda M, Fairhurst RM, et al. Spread of artemisinin resistance in Plasmodium falciparum malaria. N Engl J Med. 2014;371(5):411-423. https://doi.org/10.1056/NEJMoa1314981

13. Uwimana A, Legrand E, Stokes BH, et al. Emergence and clonal expansion of in vitro artemsinin-resistant Plasmodium falciparum kelch13R561H mutant parasites in Rwanda. Nature Med 2020;26:1602-1608. https://doi.org/10.1038/s41591-020-1005-2

14. Barnes KI, Durrheim DN, Little F, et al. Effect of artemether-lumefantrine policy and improved vector control on malaria burden in KwaZulu-Natal, South Africa. PLoS Med 2005;2(11):e330. https://doi. org/10.1371/journal.pmed.0020330

15. Raman J, Allen E, Workman L, et al. Safety and tolerability of single low-dose primaquine in a lowintensity transmission area in South Africa: An open-label, randomized controlled trial. Malar J intensity transmission area in South Africa: An open-
2019;18:209. https://doi.org/10.1186/s12936-019-2841-8

16. Ashley EA, Stepniewska K, Lindegardh N, et al. How much fat is necessary to optimize lumefantrine 16. Ashley EA, Stepniewska K, Lindegardh N, et al. How much fat is necessary to optimize lumefantrine
oral bioavailability? Trop Med Int Health 2007;12(2):195-200. https://doi.org/10.1111/j.13653156.2006 .01784
17. Kloprogge F, Workman L, Borrmann S, et al. Artemether-lumefantrine dosing for malaria treatment in Kloprogge F, Workman L, Borrmann S, et al. Artemether-lumefantrine dosing for malaria treatment in
young children and pregnant women: A pharmacokinetic-pharmacodynamic meta-analysis. PLoS Med young children and pregnant women: A pharmacokinetic-pharmacody

18. Chotsiri P, Denoeud-Ndam L, Baudin E, et al. Severe acute malnutrition results in lower lumefantrine exposure in children treated with artemether-lumefantrine for uncomplicated malaria. Clin Pharmacol Ther 2019;106(6):1299-1309. https://doi.org/10.1002/cpt.1531

19. Francis J, Barnes KI, Workman L, et al. An individual participant data population pharmacokinetic meta-analysis of drug-drug interactions between lumefantrine and commonly used antiretroviral treatment. Antimicrob Agents Chemother 2020;64(5):e02394-19. https://doi.org/10.1128/AAC.02394-19

20. Lamorde M, Byakika-Kibwika P, Mayito J, et al. Lower artemether, dihydroartemisinin and lumefantrine concentrations during rifampicin-based tuberculosis treatment. AIDS 2013;27(6):961-965. https://doi. org/10.1097/QAD.0b013e32835cae3b

21. World Health Organization. Updated WHO Policy Recommendation: Single dose primaquine as a gametocytocide in Plasmodium falciparum malaria. October 2012. https://www.who.int/malaria/ publications/atoz/who_pq_policy_recommendation/en/ (accessed 25 October 2020).

22. Bastiaens GJH, Tiono AB, Okebe J, et al. Safety of single low-dose primaquine in glucose-6-phosphate . Bastiaens $\mathrm{GJH}$, Tiono AB, Okebe J, et al. Safety of single low-dose primaquine in glucose-6-phosphate
dehydrogenase deficient falciparum-infected African males: Two open-label, randomised, safety trials. PLoS ONE 2018;13(1):e0190272. https://doi.org/10.1371/journal.pone.0190272

23. World Health Organization. Guidelines for the Treatment of Malaria. 3rd ed. Geneva: WHO, 2015. https://apps.who.int/iris/bitstream/handle/10665/162441/9789241549127_eng.pdf (accessed 25 October 2020).

24. Sinclair D, Donegan S, Isba R, et al. Artesunate versus quinine for treating severe malaria. Cochrane Database Syst Rev 2012, Issue 6. Art. No.: CD005967. https://doi.org/10.1002/14651858.CD005967.pub4

25. Zoller $\mathrm{T}$, Junghanass $\mathrm{T}$, Kapaun $\mathrm{A}$, et al. Intravenous artesunate for severe malaria in travellers, Europe Emerg Infect Dis 2011;17(5):771-777. https://doi.org/10.3201/eid1705.101229

26. Fried M, Duffy PE. Malaria during pregnancy. Cold Spring Harb Perspect Med 2017;7:a025551. https:// doi.org/10.1101/chhperspect.a025551

27. Saito M, Mansoor R, Kennon K, et al. Efficacy and tolerability of artemisinin-based and quinine-based treatments for uncomplicated falciparum malaria in pregnancy: A systematic review and individual patient data meta-analysis. Lancet Infect. Dis 2020;20(8):943-952. https://doi.org/10.1016/S14733099(20)30064-5

28. National Department of Health, South Africa. National guidelines for the treatment of malaria, South Africa 2019. https://www.nicd.ac.za/wp-content/uploads/2017/03/National-Guidelines-for-MalariaTreatment-SEPTEMBER-2019-Update-WITH-FRONT.pdf (accessed 25 October 2020).

29. Looareesuwan S, Phillips RE, White NJ, et al. Quinine and severe falciparum malaria in late pregnancy. Lancet 1985;2(8445):4-8. https://doi.org/10.1016/s0140-6736(85)90056-x

30. National Department of Health, South Africa. Rapid review of tocilizumab for COVID-19. 15 April 2020. http://www.health.gov.za/index.php/national-essential-medicine-list-committee-nemlc/category/633covid-19-rapid-reviews?download=4137:rapid-review-of-tocilizumab-for-covid-19 (accessed 25 October 2020).

31. National Department of Health, South Africa. Rapid review of chloroquine prophylaxis for COVID-19. 18 June 2020. http://www.health.gov.za/index.php/national-essential-medicine-list-committee-nemlc/ category/633-covid-19-rapid-reviews?download=4380:rapid-review-of-chloroquine-prophylaxis-forcovid-19-18june2020 (accessed 25 October 2020)

32. National Department of Health, South Africa. Clinical management of suspected or confirmed COVID-19 disease: Version 4 (18th May 2020). https://www.nicd.ac.za/wp-content/uploads/2020/05/ Clinical-management-of-suspected-or-confirmed-COVID-19-Version-4.pdf (accessed 25 October 2020)

33. Infante M, Ricordi C, Alejandro R, et al. Hydroxychloroquine in the COVID-19 pandemic era: In pursuit of a rational use for prophylaxis of SARS-CoV-2 infection. Expert Rev Anti Infect Ther 2020 (epub 16 August 2020). https://doi.org/10.1080/14787210.2020.1799785

34. Liu J, Cao R, Xu M, et al. Hydroxychloroquine, a less toxic derivative of chloroquine, is effective in inhibiting SARS-CoV-2 infection in vitro. Cell Discov 2020;6:16. https://doi.org/10.1038/s41421-
ing 020-0156-0

35. Mitja O, Corbacho-Monne M, Ubals M, et al. Hydroxychloroquine for early treatment of adults with mild COVID-19: A randomised-controlled trial. Clin Infect Dis 2020;ciaa1009. https://doi.org/10.1093/ $\mathrm{cid} / \mathrm{ciaa} 1009$

36. Geleria J, Sun Y, Platt J, et al. Observational study of hydroxychloroquine in hospitalised patients with COVID-19. N Engl J Med 2020;382:2411-2418. https://doi.org/10.1056/NEJMoa2012410

37. Rosenberg ES, Dufort EM, Udo T, et al. Association of treatment with hydroxychloroquine or azithromycin with in-hospital mortality in patients with COVID-19 in New York State. JAMA 2020;323(24):2493-2502. https://doi.org/10.1001/jama.2020.8630

38. Michaud V, Dow P, Al Rihani SB, et al. Risk of drug-induced long QT syndrome associated with the use of repurposed COVID-19 drug: A systematic review. medRxiv 2020 (epub 2 September 2020). https:// doi.org/10.1101/2020.04.21.20066761

39. Singer ME, Kaelber CD, Antonelli MJ. Hydroxychloroquine ineffective for COVID-19 prophylaxis in lupus and rheumatoid arthritis. Ann Rheum Dis 2020 (epub 5 August 2020). https://doi.org/10.1136/ annrheumdis-2020-218500

40. World Health Organization. Model list of essential medicines. 2020. https://www.who.int/groups/ expert-committee-on-selection-and-use-of-essential-medicines/essential-medicines-lists (accessed 25 October 2020).

41. Payne D. Spread of chloroquine resistance in Plasmodium falciparum. Parasitol Today 1987;3(8):214-246. https://doi.org/10.1016/0169-4758(87)90147-5

42. Efferth T. Molecular pharmacology and pharmacogenomics of artemisinin and its derivatives in cancer cells. Curr Drug Targets 2006;7(4):407-421. https://doi.org/10.2174/138945006776359412 